\title{
BMC Neuroscience reviewer acknowledgement 2014
}

Tim Shipley

\section{Contributing reviewers}

The editors of BMC Neuroscience would like to thank all our reviewers who have contributed to the journal in Volume 15 (2014).

Paula Agostinho
Portugal

Saif Ahmad Saudi

Arabia

Ludwig Aigner

Austria

Michael Akins

United States of America

Hakan Aldskogius

Sweden

John Allen

United States of America

Silvana Allodi

Brazil

Julià L Amengual

Spain

Miroslava Anderova

Czech Republic

Balasubramaniam Annamalai

United States of America

\section{Saema Ansar}

Sweden

Ubaldo Armato

Italy

Mariana Astiz

Spain

\section{Ilknur Ay}

United States of America

Travis Baker

Canada

Veera Venkata Ratnam Bandaru

United States of America

Rina Bandopadhyay

United Kingdom

Andrew Bankston

United States of America

Jianxin Bao

United States of America

Guido Barchiesi

Italy

Anirban Basu

India

Nadhim Bayatti

United Kingdom

Richard Bazinet

United States of America

M. Flint Beal

United States of America

Maik Behrens

Germany

Christian Bellebaum

Germany
Gian Carlo Bellenchi

Italy

Kerstin Bendfeldt

Switzerland

Itai Berger

Israel

Sablonniere Bernard

France

Neda Bernasconi

Canada

Hans-Gert Bernstein

Germany

John Bethea

United States of America

Pallab Bhattacharya

India

Knut Biber

Germany

Patrick Blader

France

Nico Boehler

United States of America

Corina Bondi

United States of America

Cesar Borlongan

United States of America

Correspondence: tim.shipley@biomedcentral.com

BioMed Central, Floor 6, 236 Gray's Inn Road, London WC1X 8HB, UK 
Marco Bortolato

United States of America

Yvonne Böttcher

Germany

Matthew Boyko

Israel

Patrick Bradshaw

United States of America

Clive Bramham

Norway

Roland Brandt

Germany

Filippo Brighina

Italy

Guy Brock

United States of America

Brad Broughton

Australia

Emmanuel Brouillet

France

Russell Brown

United States of America

Anna Bukiya

United States of America

Nico Bunzeck

Germany

Malgorzata Burek

Germany

Josh Burk

United States of America

Nikita Burke

Ireland

Kimberly Byrnes

United States of America

Laura Calza

Italy

Joshua Cameron

United States of America

Antoni Camins

Spain

Simona Capsoni

Italy

Filippo Caraci

Italy

\begin{tabular}{|c|c|}
\hline $\begin{array}{l}\text { Marina Cardano } \\
\text { Italy }\end{array}$ & $\begin{array}{l}\text { Peter De Lissa } \\
\text { Australia }\end{array}$ \\
\hline $\begin{array}{l}\text { Thierry Charlier } \\
\text { United States of America }\end{array}$ & $\begin{array}{l}\text { Amanda De Oliveira } \\
\text { Brazil }\end{array}$ \\
\hline $\begin{array}{l}\text { Aiqing Chen } \\
\text { United Kingdom }\end{array}$ & $\begin{array}{l}\text { Cecile Delarasse } \\
\text { France }\end{array}$ \\
\hline $\begin{array}{l}\text { Huafu Chen } \\
\text { China }\end{array}$ & $\begin{array}{l}\text { Cinzia Dello Russo } \\
\text { Italy }\end{array}$ \\
\hline $\begin{array}{l}\text { Sidi Chen } \\
\text { United States of America }\end{array}$ & $\begin{array}{l}\text { Catherine Dermon } \\
\text { Greece }\end{array}$ \\
\hline $\begin{array}{l}\text { Sk Cheong } \\
\text { Malaysia }\end{array}$ & $\begin{array}{l}\text { Stéphanie Deroo } \\
\text { Belgium }\end{array}$ \\
\hline $\begin{array}{l}\text { Glyn Chidlow } \\
\text { Australia }\end{array}$ & $\begin{array}{l}\text { Paula Desplats } \\
\text { United States of America }\end{array}$ \\
\hline $\begin{array}{l}\text { Shinung Ching } \\
\text { United States of America }\end{array}$ & $\begin{array}{l}\text { Megan Detloff } \\
\text { United States of America }\end{array}$ \\
\hline $\begin{array}{l}\text { Andrew Chisholm } \\
\text { United States of America }\end{array}$ & $\begin{array}{l}\text { Tajinder Dhammu } \\
\text { United States of America }\end{array}$ \\
\hline $\begin{array}{l}\text { Byung Tae Choi } \\
\text { Korea, South }\end{array}$ & $\begin{array}{l}\text { Narender Dhingra } \\
\text { India }\end{array}$ \\
\hline $\begin{array}{l}\text { Winyoo Chowanadisai } \\
\text { United States of America }\end{array}$ & $\begin{array}{l}\text { James Dillman } \\
\text { United States of America }\end{array}$ \\
\hline $\begin{array}{l}\text { Tumul Chowdhury } \\
\text { Canada }\end{array}$ & $\begin{array}{l}\text { Dragan Djuric } \\
\text { Serbia }\end{array}$ \\
\hline $\begin{array}{l}\text { Sylvie Claeysen } \\
\text { France }\end{array}$ & $\begin{array}{l}\text { Robert Donati } \\
\text { United States of America }\end{array}$ \\
\hline $\begin{array}{l}\text { Marc Claret } \\
\text { Spain }\end{array}$ & $\begin{array}{l}\text { Sarah Donohue } \\
\text { United States of America }\end{array}$ \\
\hline $\begin{array}{l}\text { David Clayton } \\
\text { United Kingdom }\end{array}$ & $\begin{array}{l}\text { Ayse Dosemeci } \\
\text { United States of America }\end{array}$ \\
\hline $\begin{array}{l}\text { Luciano Conti } \\
\text { Italy }\end{array}$ & $\begin{array}{l}\text { Rose Du } \\
\text { United States of America }\end{array}$ \\
\hline $\begin{array}{l}\text { Richard Coppola } \\
\text { United States of America }\end{array}$ & $\begin{array}{l}\text { Dan Dumitrascu } \\
\text { Romania }\end{array}$ \\
\hline $\begin{array}{l}\text { Adam Paul Croft } \\
\text { United Kingdom }\end{array}$ & $\begin{array}{l}\text { Valentina Echeverria } \\
\text { United States of America }\end{array}$ \\
\hline $\begin{array}{l}\text { Li Dai } \\
\text { United States of America }\end{array}$ & $\begin{array}{l}\text { James Christopher Edgar } \\
\text { United States of America }\end{array}$ \\
\hline $\begin{array}{l}\text { Radhouane Dallel } \\
\text { France }\end{array}$ & $\begin{array}{l}\text { Ruben Eggers } \\
\text { Netherlands }\end{array}$ \\
\hline $\begin{array}{l}\text { Andrew Davies } \\
\text { United Kingdom }\end{array}$ & $\begin{array}{l}\text { Andrew Elliot } \\
\text { United States of America }\end{array}$ \\
\hline $\begin{array}{l}\text { Max De Leeuw } \\
\text { Netherlands }\end{array}$ & $\begin{array}{l}\text { James Eubanks } \\
\text { Canada }\end{array}$ \\
\hline
\end{tabular}

Amanda De Oliveira

Brazil

Cecile Delarasse

Cinzia Dello Russo

Greece

Stéphanie Deroo

Belgium

Paula Desplats

Megan Detloff

United States of America

Tajinder Dhammu

Narender Dhingra

James Dillman

United States of America

Dragan Djuric

Robert Donati

Sarah Donohue

United States of America

Ayse Dosemeci

of America

Rose Du

Dan Dumitrascu

Romania

Valentina Echeverria

States of America

James Christopher Edgar

Canada 
Jim Fadel

United States of America

Antonio Faiella

Italy

Tracy Farr

Germany

Mauro Fasano

Italy

S Hossein Fatemi

United States of America

Francisco Jose Fernandez Gomez

France

Isidro Ferrer
Spain

Caleb Finch

United States of America

Esther Florin

Germany

Steven Frucht

United States of America

Bozena Gabryel

Poland

Ricardo Galhardoni

Brazil

Frederick Gallun

United States of America

Olga Lucía Gamboa Arana

Germany

Leontien Geven

Netherlands

Othman Ghribi

United States of America

Alain Ghysen

France

Rashid Giniatullin

Finland

De Sarro Giovambattista

Italy

Massimo Girelli

Italy

Anna Göbel

Germany

Robert Göder

Germany
Martin Goettlich

Germany

Giorgio Gorini

United States of America

Laura Grabel

United States of America

Jessica Green

United States of America

Joel Greenberg

United States of America

Elke Griesmaier

Austria

David Hampson

Canada

Jörg Hanrieder

Sweden

Jakob Hansen

Denmark

Thalía Harmony

Mexico

Joseph Harris

Germany

Gesa Hartwigsen

Germany

Steffen Harzsch

Germany

Satoru Hayasaka

United States of America

Shuhan He

United States of America

Ying He

United States of America

Geoff Head

Australia

Terry Hebert

Canada

Mike Heilemann

Germany

Marcus Heldmann

Germany

Stefan Heller

United States of America

Niels Hellings

Belgium
Istvan Hernadi

Hungary

Johannes Hewig

Germany

Chindo Hicks

United States of America

Jens Hjerling Leffler

Sweden

Jihane Homman-Ludiye

Australia

Dewen $\mathrm{Hu}$

China

Hsien-Sung Huang

United States of America

Rene Hurlemann

Germany

Miki Igarashi

United States of America

Yuzuru Ikeda

Japan

Joji Inamasu

Japan

Yasuhiro Ishihara

Japan

Masako Isokawa

United States of America

Etsuro Ito

Japan

Michael Jackson

Canada

Norbert Jausovec

Slovenia

Suresh Jesuthasan

Singapore

Meiyan Jiang

United States of America

Dirk Junghans

Switzerland

Mohamed Kabbaj

United States of America

John Kalbfleisch

United States of America

Takashi Kanda

Japan 
Sang Soo Kang

Korea, South

Ilia Karatsoreos

United States of America

Charanjit Kaur

Singapore

Amajad Kazi

India

Mohammad Moshahid Khan

United States of America

Rajesh Khanna

United States of America

June Sic Kim

Korea, South

Jieun Kim

United States of America

Yosuke Kita

Japan

Katrin Klebermaß-Schrehof

Austria

Anthony Kline

United States of America

Ulrike Krämer

Germany

Stefanie Kuerten

Germany

Yu-Min Kuo

Taiwan

Charalambos Kyriacou

United Kingdom

Madepalli Lakshmana

United States of America

Thomas Lanz

United States of America

Robert Ledeen

United States of America

Soon-Tae Lee

United States of America

Patrick Lewis

United Kingdom

Bingcang Li

China

Yonggang Li

United States of America
Stefan Liebau

Germany

Monika Liguz-Lecznar

Poland

Weihong Lin

United States of America

Eng-Ang Ling

Singapore

Jonathan List

Germany

Hao Liu

United States of America

Guangming Lu

China

Fang Luo

China

Wenbo Luo

China

Wenyu Luo

United States of America

Svetlana Lutsenko

United States of America

Marzena Mackowiak

Poland

Mark Majchrzak

United States of America

David Male

United Kingdom

Volker Mall

Germany

Ml Mansego

Spain

Serge Marbacher

Switzerland

Paula Martin

United States of America

Mathew Martin-Iverson

Australia

Hirofumi Maruyama

Japan

Tessa Marzi

Italy

Eliezer Masliah

United States of America
Colin Masters

Australia

Fumiyo Matsuda

Japan

Gianluca Matteoli

Belgium

Gianluigi Mazzoccoli

Italy

Laura Medina-Ceja

Mexico

Suresh Mehta

United States of America

Dies Meijer

Netherlands

Onno Meijer

Netherlands

Noam Meiri

Israel

Mareike Menz

Germany

Francisco Mercado

Spain

David Miller

United States of America

Kenneth Miller

United States of America

Richard Milner

United States of America

Teresa Mitchell

United States of America

Seiji Miyata

Japan

Sumiko Mochida

Japan

Matthias Mölle

Germany

Julio Cesar Morales Medina

Mexico

Gabriela Morali

Mexico

Joe Moran

United States of America

Kyoji Morita

Japan 
Marija Mostarica-Stojkovic

Serbia

Bernadette Murphy

Canada

Shin Nagayama

United States of America

Kazuki Nagayasu

Japan

Pradeep Naik

India

Raad Nashmi

Canada

Xavier Navarro

Spain

Igor Nenadic

Germany

Winfried Neuhaus

Austria

Guilherme Neves

United Kingdom

Vadim Nikulin

Germany

Johannes Nimpf

Austria

Sharon Oneill

United States of America

Wei-Yi Ong

Singapore

Marco Onorati

United States of America

Jullie Pan

United States of America

Vinay Parikh

United States of America

Surojit Paul

United States of America

Rosetta Pedotti

Italy

Louis Penning

Netherlands

Isabel Perez Otaño

Spain

Stefanie Peykarjou

Germany
Dzung Pham

United States of America

Linda Phillips

United States of America

Emmanuel Pinteaux

United Kingdom

Paulo Pires

United States of America

Justin Piro

United States of America

Cristina Porras

Spain

Christine Portfors

United States of America

Shuhong Qiao

United States of America

Guoping Qing

China

Mario Quarantelli

Italy

Nidia Quillinan

United States of America

Monika Rabenstein

Germany

Jacob Raber

United States of America

Peter Racay

Slovakia

Biserka Radosevic-Stasic

Croatia

Valeria Ramaglia

Netherlands

Murali Ramanathan

United States of America

Cedric Raoul

France

P. Hemachandra Reddy

United States of America

Eva Redei

United States of America

George Reeke

United States of America

Lina Reiss

United States of America
Renate Reniers

United Kingdom

Mathias Rhein

Germany

Alfredo Ribeiro-Da-Silva

Canada

Jeremy Rich

United States of America

William Richardson

United Kingdom

Ulrike Rimmele

Switzerland

Maja Rogic

Croatia

Patricia Rojas

Mexico

Mikael Roll

Sweden

Alejandro Romero

Spain

Aribert Rothenberger

Germany

Eugeni Roura

Australia

Ruth Ruscheweyh

Germany

Gesine Saher

Germany

Manabu Sakakibara

Japan

Harutoshi Sakakima

Japan

Dan Sanes

United States of America

Kazunori Sango

Japan

Kazunobu Sawamoto

Japan

Mohammad Sayyah

Iran

Mya Schiess

United States of America

Felix Schlachetzki

Germany 
Matthias Schlesewsky

Germany

Alexander Schmidt

Germany

Leonid Schneider

Germany

Mircea Ariel Schoenfeld

Germany

Stefanie Schreiber

Germany

Markus Schubert

Germany

Enrico Schulz

United Kingdom

Sarah Schuster

Austria

Karen Scott

Ireland

Tommy Seaborn

Canada

Nicolas Sergeant

France

Bridget Shafit-Zagardo

United States of America

Zahoor Shah

United States of America

Rebecca Shansky

United States of America

Anandakumar Shunmugavel

United States of America

Bai Chuang Shyu

Taiwan

James Sikela

United States of America

Luigi Sironi

Italy

David Slattery

Germany

Robert Smith

United States of America

Po-Wah So

United Kingdom

Wesley Solomon

United States of America
Enrique Soto

Mexico

Kim Staats

Belgium

Mark Stahl

United States of America

Jeannette Stankowski

United States of America

Axel Steiger

Germany

Ivana Stevanovic

Serbia

Jerry Stitzel

United States of America

Judith Strong

United States of America

Aideen Sullivan

Ireland

Joanna Sypecka

Poland

Gabor Szabo

Hungary

Changiz Taghibiglou

Canada

Saeid Taheri

United States of America

Nobuyuki Takei

Japan

Andrew Tapper

United States of America

Silke Telkemeyer

Germany

Charlotte Teunissen

Netherlands

Wipawan Thangnipon

Thailand

Ronald Thomas

United States of America

Christopher Thompson

United States of America

Veronika Thorns

Germany

Lixia Tian

China
Xuebi Tian

China

Piotr Topilko

France

Germán Torregrosa

Spain

Pablo Torterolo

Uruguay

Joseph Tracy

United States of America

Richa Tripathi

United Kingdom

Vladimir Tsibulsky

United States of America

Oliver Tuescher

Germany

Kwong-Chung Tung

Taiwan

Bradley Turner

Australia

Ann Turnley

Australia

Henning Ulrich

Brazil

Elias Utreras

Chile

Fred Van Leuven

Belgium

Marie-José Van Tol

Netherlands

Alessandro Vercelli

Italy

Marjolein Verly

Belgium

Matthijs Vink

Netherlands

Logan Voss New

Zealand

Pascal Vrticka

United States of America

Esther Walton

Germany

Chao Wang

United States of America 
Chengzhong Wang

United States of America

Dan Wang

Japan

\section{Gang Wang}

United States of America

Tongfei Wang

United States of America

Xiaoxi Wang

United States of America

Tracy Warbrick

Germany

Katrin Willig

Germany

David Wirtshafter

United States Minor Outlying Islands

Yung Hou Wong

China

Kin Foon Kevin Wong

United States of America
Eligiusz Wronka

Poland

Ching Hsiang Wu

Taiwan

Anhua Wu

China

Xu Xue-Bing

China

Ipek Yalcin Christmann

France

Kenji Yamamoto

Japan

Yongjie Yang

United States of America

Yongquan Ye

United States of America

Shan Ping Yu

United States of America

Zengqiang Yuan

China
Ariane Zamoner

Brazil

Xia Zhang

Canada

Yumin Zhang

United States of America

Deming Zhao

China

Hongling Zhu

United States of America

Lei Zhu

United States of America

Ling-Qiang Zhu

China

Xi-Nian Zuo

China 